\title{
Interfacial Anchor Properties of Tryptophan Residues in Transmembrane Peptides Can Dominate over Hydrophobic Matching Effects in Peptide-Lipid Interactions ${ }^{\dagger}$
}

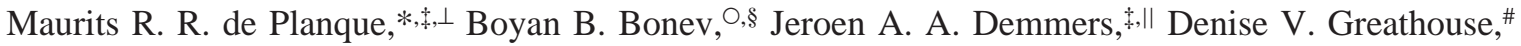 \\ Roger E. Koeppe II, ${ }^{\#}$ Frances Separovic, ${ }^{\otimes}$ Anthony Watts, ${ }^{\S}$ and J. Antoinette Killian,, \\ Department of Biochemistry of Membranes, Center for Biomembranes and Lipid Enzymology, \\ Institute of Biomembranes, Utrecht University, Padualaan 8, 3584 CH Utrecht, The Netherlands, \\ Biomembrane Structure Unit, Biochemistry Department, Oxford University, Oxford OX1 3QU, United Kingdom, \\ Department of Biomolecular Mass Spectrometry, Bijvoet Center for Biomolecular Research and Utrecht Institute for \\ Pharmaceutical Sciences, Utrecht University, Sorbonnelaan 16, 3584 CA Utrecht, The Netherlands, \\ Department of Chemistry and Biochemistry, University of Arkansas, Fayetteville, Arkansas 72701, and \\ School of Chemistry, University of Melbourne, Melbourne, VIC 3010, Australia
}

Received October 14, 2002; Revised Manuscript Received February 7, 2003

\begin{abstract}
Membrane model systems consisting of phosphatidylcholines and hydrophobic $\alpha$-helical peptides with tryptophan flanking residues, a characteristic motif for transmembrane protein segments, were used to investigate the contribution of tryptophans to peptide-lipid interactions. Peptides of different lengths and with the flanking tryptophans at different positions in the sequence were incorporated in relatively thick or thin lipid bilayers. The organization of the systems was assessed by NMR methods and by hydrogen/deuterium exchange in combination with mass spectrometry. Previously, it was found that relatively short peptides induce nonlamellar phases and that relatively long analogues order the lipid acyl chains in response to peptide-bilayer mismatch. Here it is shown that these effects do not correlate with the total hydrophobic peptide length, but instead with the length of the stretch between the flanking tryptophan residues. The tryptophan indole ring was consistently found to be positioned near the lipid carbonyl moieties, regardless of the peptide-lipid combination, as indicated by magic angle spinning NMR measurements. These observations suggest that the lipid adaptations are not primarily directed to avoid a peptide-lipid hydrophobic mismatch, but instead to prevent displacement of the tryptophan side chains from the polar-apolar interface. In contrast, long lysine-flanked analogues fully associate with a bilayer without significant lipid adaptations, and hydrogen/deuterium exchange experiments indicate that this is achieved by simply exposing more (hydrophobic) residues to the lipid headgroup region. The results highlight the specific properties that are imposed on transmembrane protein segments by flanking tryptophan residues.
\end{abstract}

Three-dimensional structures of integral membrane proteins generally show distinct belts of aromatic and charged residues on both sides of the membrane-spanning segments $(1-5)$. In line with this observation, analyses of protein sequence data indicate that the predominantly hydrophobic

† This work was supported by the Council for Chemical Sciences (CW) with financial aid from The Netherlands Organization for Scientific Research (NWO), by EMBO Fellowship ASTF 8778 (to M.R.R.deP.), by EU TMR Network ERBFMRX-CT96-0004, by NIH Grant GM 34968 (to R.E.K. and D.V.G.), and by NATO Grant CRG 950357.

* Corresponding authors (e-mail m.r.r.deplanque@chem.uu.nl or j.a.killian@chem.uu.nl).

Center for Biomembranes and Lipid Enzymology, Institute of Biomembranes.

${ }^{\perp}$ Present address: Department of NMR Spectroscopy, Bijvoet Center for Biomolecular Research, Utrecht University, Padualaan 8, $3584 \mathrm{CH}$ Utrecht, The Netherlands.

${ }^{\circ}$ Present address: School of Biomedical Sciences, University of Nottingham, Nottingham, NG7 2UH, United Kingdom.

$\S$ Oxford University.

" Bijvoet Center for Biomolecular Research and Utrecht Institute for Pharmaceutical Sciences.

\# University of Arkansas.

${ }^{\otimes}$ University of Melbourne. stretches of $\sim 20$ hydrophobic residues that represent putative transmembrane helices are preferentially flanked by aromatic and charged residues $(6,7)$. It is estimated that in membranes, the hydrophobic stretch is approximately aligned with the lipid acyl chains, and it has been proposed that it is energetically favorable for a membrane system to experience such a match between peptide hydrophobic length and bilayer hydrophobic thickness. Consequently, a tendency to avoid hydrophobic mismatch situations could play a role in membrane organization $(8-10)$. However, it has also been postulated that the flanking aromatic and charged residues, which are estimated to be in contact with the lipid carbonyl and headgroup regions, have a specific affinity for the membrane-water interface. Hence, a need to avoid exposure of these residues to either too hydrophobic or too polar bilayer regions could also contribute to peptide/lipid interactions $(11-14)$.

Model membrane systems consisting of phospholipids and synthetic transmembrane peptides have been used extensively to address the relationship between hydrophobic matching and membrane organization $(9,15-19)$. We have shown previously that polyleucine-alanine peptides that are flanked 
on both sides by tryptophan residues can significantly influence the organization of phosphatidylcholine model membranes when the peptides are relatively long or short with respect to bilayer hydrophobic thickness $(9,20-23)$. However, because the Trp residues of the studied peptides are positioned at or near the termini of the peptides, hydrophobic mismatch also implies that these residues become exposed to a different interfacial environment. As a result, the observed membrane response to the incorporation of relatively short or long peptides could be driven not only by a tendency to avoid hydrophobic mismatch situations but also by a tendency of the flanking residues to avoid displacement from specific interfacial sites.

In the present study, we chose to investigate the extent to which a preference of Trp side chains for localization at the lipid-water interface can influence mismatch effects. For this purpose, peptides with flanking Trp residues at different positions in the peptide sequence were synthesized and were incorporated in phosphatidylcholine bilayers. The influence of these variations in peptide sequence was studied under conditions of both positive and negative mismatch. In addition, the locations of Trp residues within the lipid bilayer were investigated for peptides of various lengths. The results indicate that the membrane rearrangements induced by Trpflanked peptides are driven by the propensity to position the Trp residues at a well-defined preferred interfacial location, and not primarily by a tendency to avoid hydrophobic mismatch. Such behavior was not observed for Lys-flanked peptides, which were included in this study for comparison. The results give experimental support to the concept that Trp residues can act as interfacial membrane anchors and that interfacial interactions can contribute significantly to membrane organization.

\section{MATERIALS AND METHODS}

\section{Materials}

All phospholipids were purchased from Avanti Polar Lipids Inc. (Birmingham, AL). WALP ${ }^{1}$ and KALP peptides were synthesized and analyzed by analytical HPLC and electrospray mass spectrometry as described previously (22, 24). Peptide purity was found to be $>90 \%$ according to analytical HPLC. The identity of the peptides was confirmed by electrospray mass spectrometry.

\section{Methods}

Sample Preparation. WALP peptides were quantified by the absorbance of tryptophan at $280 \mathrm{~nm}$ (using a molar extinction coefficient of $5600 \mathrm{M}^{-1} \mathrm{~cm}^{-1}$ for each Trp residue) and KALP analogues by their dry weight. Unless stated otherwise, samples were prepared in the following manner. Peptides were dissolved in $10 \mu \mathrm{L}$ of trifluoroacetic acid/mg of peptide and, after evaporation of the solvent by a stream of nitrogen, were redissolved in $1 \mathrm{~mL}$ of trifluoroethanol,

\footnotetext{
${ }^{1}$ Abbreviations: CD, circular dichroism; NMR, nuclear magnetic resonance; MAS, magic angle spinning; MS, mass spectrometry; H/D, hydrogen/deuterium; ESI, electrospray ionization; Etn, ethanolamine; Ac, acetyl; Tris, tris(hydroxymethyl)aminomethane; WALP, tryptophanalanine-leucine peptide; KALP, lysine-alanine-leucine peptide; PC, phosphatidylcholine; 14:0-PC, 1,2-dimyristoyl-sn-glycero-3-phosphocholine; 18:1 1 -PC, 1,2-dioleoyl-sn-glycero-3-phosphocholine; $\mathrm{L}_{\alpha}$, liquid crystalline bilayer phase; $\mathrm{I}$, isotropic phase; $\mathrm{H}_{\mathrm{II}}$, inverse hexagonal phase.
}

followed by drying in a rotary evaporator. This step was repeated once. Next, the peptides were dissolved in $0.5 \mathrm{~mL}$ of trifluoroethanol and added to $0.5 \mathrm{~mL}$ of a lipid suspension in distilled water, at temperatures above the gel to liquid crystalline phase transition, followed by addition of $10 \mathrm{~mL}$ of water and immediate lyophilization. Lipid-peptide mixtures were hydrated in buffer $[100 \mathrm{mM} \mathrm{NaCl}$ and $25 \mathrm{mM}$ Tris ( $\mathrm{pH} 7.4)]$ or in distilled water and were subsequently centrifuged $\left(30000 \mathrm{~g}, 15 \mathrm{~min}, 4^{\circ} \mathrm{C}\right)$, and the supernatant was removed. Pellets were washed if the $\mathrm{pH}$ of the supernatant was $<6$. Next, the pellets were resuspended in $200 \mu \mathrm{L}$ of buffer or distilled water and subjected to 10 freeze-thaw cycles to equilibrate the sample.

Circular Dichroism Spectroscopy. Peptide-lipid mixtures with a 1:30 peptide/lipid molar ratio (containing $7.5 \mu \mathrm{mol}$ of lipid) were prepared as described above and were hydrated in $1 \mathrm{~mL}$ of buffer. The samples was then sonicated $(5 \mathrm{~min}$, $50 \%$ duty cycle, input power of $40 \mathrm{~W}$, Branson 250 tip sonicator). To pellet down titanium particles and any residual multilamellar lipid structures, the sonicated sample was next centrifuged $\left(30000 \mathrm{~g}, 15 \mathrm{~min}, 4^{\circ} \mathrm{C}\right)$. CD measurements were carried out on a Jasco J-600 spectropolarimeter as described previously $(22,25)$. CD spectra of sonicated vesicles in excess water were obtained to assess the helicity of the peptide, and spectra of oriented peptide-lipid films were used to confirm a transmembrane peptide topology. CD spectra of pure peptides in water were recorded $(22,25)$ and confirmed that only the peptide population that is bilayerincorporated contributed to the CD signal.

${ }^{31}$ P Nuclear Magnetic Resonance Measurements. Samples with a 1:10 peptide/lipid molar ratio (containing $20 \mu \mathrm{mol}$ of lipid), hydrated in $1.5 \mathrm{~mL}$ of buffer, were measured on a Bruker MSL300 NMR spectrometer. The sample temperature was regulated at $30{ }^{\circ} \mathrm{C}$ using a Bruker B-VT1000 temperature controller. Proton-decoupled experiments were carried out at $121.5 \mathrm{MHz}\left(17 \mu \mathrm{s} 90^{\circ}\right.$ pulse, $1.3 \mathrm{~s}$ interpulse time, gated proton-noise decoupling). Approximately 15000 scans were acquired, and a sweep width of $25 \mathrm{kHz}$ and 1024 complex data points were used. Prior to Fourier transformation, an exponential multiplication was applied, resulting in a 100 $\mathrm{Hz}$ line broadening. The chemical shift was referenced to a sample with isotropically moving PC molecules. All spectra were scaled to the same height.

${ }^{2} \mathrm{H}$ Nuclear Magnetic Resonance Measurements and Analysis. Spectra of samples containing peptide and $15 \mu \mathrm{mol}$ of $s n$-2 perdeuterated 14:0-PC at a 1:30 molar ratio, hydrated in $1 \mathrm{~mL}$ of deuterium-depleted water, were recorded on a Varian Unity400+ NMR spectrometer, using a broadband probe with a $10 \mathrm{~mm}$ solenoidal sample coil. ${ }^{2} \mathrm{H}$ NMR measurements were performed at $61.4 \mathrm{MHz}$ and at $34^{\circ} \mathrm{C}$ as previously described (25), and resulting unsymmetrized ${ }^{2} \mathrm{H}$ NMR powder spectra were analyzed by the dePakeing procedure (20). This procedure results in spectra that would be obtained for an aligned membrane with its bilayer normal parallel to the magnetic field and enhances the resolution of the spectral doublets with splittings $\Delta v_{\mathrm{Q}}$ that relate to the segmental order parameter $S(i)$. Order parameter profiles were obtained by assuming that the segmental order varies monotonically along the acyl chain from the $\mathrm{C}^{3} \mathrm{D}_{2}$ to the $\mathrm{C}^{14} \mathrm{D}_{3}$ moiety. From the average order parameter, the effective length of the acyl chains was estimated and extrapolated to the hydrophobic bilayer thickness as de- 
scribed previously (20). Such an extrapolation implies that the average order parameter is directly correlated to the hydrophobic bilayer thickness. It should be noted that this assumption is not supported, however, by $\mathrm{X}$-ray studies carried out on oriented WALP/PC systems at lower water content (26). Nevertheless, the ${ }^{2} \mathrm{H}$ NMR-derived average order parameter is a very sensitive indicator of peptideinduced acyl chain ordering, and estimated effects on bilayer thickness are a useful parameter to compare the influence of the different peptides.

${ }^{1}$ H Magic Angle Spinning Nuclear Magnetic Resonance Measurements. Samples were prepared by mixing peptide in trifluoroethanol and lipid in methanol, followed by drying in a rotary evaporator and under high vacuum. The dried lipid-peptide films with a 1:10 peptide/lipid molar ratio (containing $30 \mu \mathrm{mol}$ of lipid) were hydrated in $1.5 \mathrm{~mL}$ of $\mathrm{D}_{2} \mathrm{O}$, vigorously vortexed, and subjected to 10 freeze-thaw cycles to equilibrate the sample. The resulting liposome suspensions were centrifuged $(30000 \mathrm{~g}, 15 \mathrm{~min})$, after which excess $\mathrm{D}_{2} \mathrm{O}$ was removed. Approximately $10 \mu \mathrm{mol}$ of the lipid dispersion was transferred to a 4-mm rotor, and ${ }^{1} \mathrm{H}$ MAS NMR spectra were subsequently recorded on a Bruker Avance 400 spectrometer equipped with an HX Bruker probe. Measurements were carried out at $400.13 \mathrm{MHz}$, with a $5 \mu \mathrm{s} 90^{\circ}$ pulse and a $2.0 \mathrm{~s}$ interpulse time. Typically, 1024 scans were acquired at $34{ }^{\circ} \mathrm{C}$, using a sweep width of 50 $\mathrm{kHz}$ and 16384 complex data points, while the sample rotation speed was maintained at $3 \mathrm{kHz}$. No line broadening was applied prior to Fourier transformation. For the first sample measured, the water resonance was set to $4.50 \mathrm{ppm}$, and in subsequent measurements recalibration of the chemical shift was not required. Resonances were assigned according to ref 27.

Hydrogen/Deuterium Exchange and Mass Spectrometry. Sample preparation and measurements were performed as described previously $(28,29)$. Peptide solutions in trifluoroethanol $(1 \mathrm{~mL}, 0.46 \mathrm{mM})$ were added to $14: 0-\mathrm{PC}$ solutions in methanol $(1 \mathrm{~mL}, 12 \mathrm{mM})$ and vigorously vortexed. The solvent was removed by evaporation in a rotavapor, and the mixed films were then dried for $24 \mathrm{~h}$ under high vacuum. The films were hydrated in $0.5 \mathrm{~mL}$ of $10 \mathrm{mM}$ ammonium acetate buffer ( $\mathrm{pH}$ 7.5). Large unilamellar vesicles were prepared by extrusion through a $400-\mathrm{nm}$ filter at room temperature and kept at $4{ }^{\circ} \mathrm{C}$ until use. Before the start of $\mathrm{H} / \mathrm{D}$ exchange, the vesicles were preincubated at $30{ }^{\circ} \mathrm{C}$ for at least $30 \mathrm{~min}$. For accurate comparison of the exchange data, vesicle suspensions with different peptide compositions were mixed prior to the start of the exchange. Suspensions were then 50 times diluted in deuterated ammonium acetate buffer at $30{ }^{\circ} \mathrm{C}(10 \mathrm{mM}, \mathrm{pH} 7.5)$, containing $\sim 1 \mathrm{mM} \mathrm{NaI}$. At selected time points, $2 \mu \mathrm{L}$ of this diluted suspension was transferred into a gold-coated glass capillary and the measurement was started as quickly as possible, whereby different peptides were analyzed simultaneously. MS measurements were performed on a Q-Tof ESI instrument (Micromass Ltd., Manchester, U.K.) or on an LCT ESI instrument (Micromass Ltd.), both operating in positive ion mode and equipped with a Z-spray nano-ESI source. The potential between the nano-ESI capillary and the orifice of the mass spectrometer was typically set to $1800 \mathrm{~V}$, and the cone voltage was $140 \mathrm{~V}$. The nanospray needle was constantly kept at $\sim 30{ }^{\circ} \mathrm{C}$. Increases in deuterium content

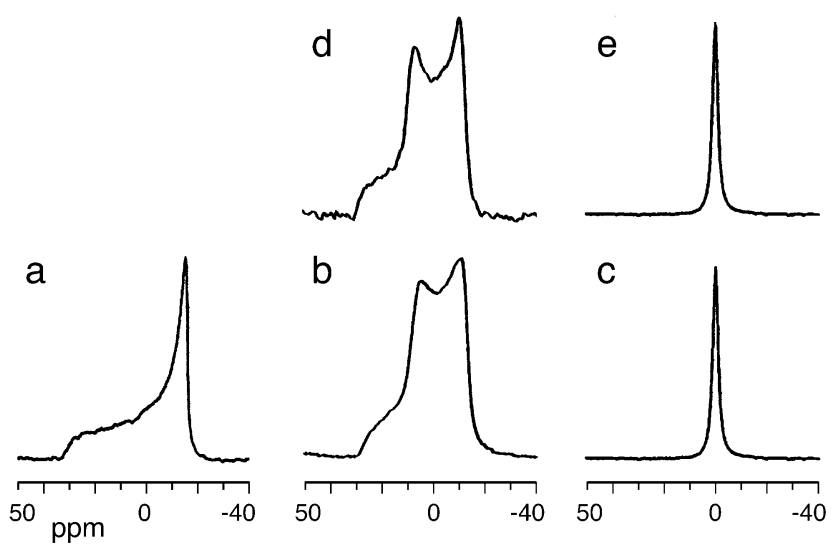

FIGURE 1: ${ }^{31} \mathrm{P}$ NMR spectra of dispersions of $18: 1_{\mathrm{c}}-\mathrm{PC}$ in the absence (a) and presence of $\mathrm{W}_{2}{ }^{\text {in ALP16 }}$ (b), $\mathrm{W}_{2}{ }^{\text {out ALP16 (c), }}$ WALP16 (d), and WALP17 (e), at a 1:10 peptide/lipid molar ratio.

(in daltons) were calculated by using the average mass-tocharge $(\mathrm{m} / \mathrm{z})$ values of the isotope clusters of the undeuterated peptides and of the partly deuterated peptides.

\section{RESULTS}

Lipid Bilayer Perturbations by Relatively Short TrpFlanked Peptides. Incorporation of relatively short transmembrane peptides, flanked by aromatic or charged residues, into PC lipid complexes may lead to the formation of nonlamellar phases, which can be readily identified by ${ }^{31} \mathrm{P}$ NMR $(21,22,25)$. As illustrated in Figure 1, pure 18:1 1 -PC gives a spectrum with a low field shoulder and a high field peak, typical for the preferred planar bilayer organization of this lipid in the liquid-crystalline $\left(\mathrm{L}_{\alpha}\right)$ phase $(30,31) .{ }^{31} \mathrm{P}$ NMR spectra of $18: 1_{\mathrm{c}}-\mathrm{PC}$ are clearly modulated by the presence of relatively short WALP peptides (Figure 1). To investigate whether it is the total length of these hydrophobic peptides or, alternatively, the inter-tryptophan distance that primarily determines which lipid phase will form, we investigated the effects of $\mathrm{W}_{2}{ }^{\text {in }} \mathrm{ALP} 16$ and $\mathrm{W}_{2}{ }^{\text {out }} \mathrm{ALP} 16$. These two analogues have the same number of hydrophobic residues as WALP16, but have fewer tryptophans and differ in the relative positions of the flanking Trp residues (Table 1). As shown in Figure 1, the $\mathrm{W}_{2}{ }^{\text {in }} \mathrm{ALP} 16$ peptide induces an $\mathrm{H}_{\text {II }}$ phase in $18: 1_{\mathrm{c}}-\mathrm{PC}$, as characterized by the spectral component with inverted asymmetry and a 2 -fold reduced chemical shift anisotropy compared to the $\mathrm{L}_{\alpha}$ spectrum (30, 31 ). However, the $\mathrm{W}_{2}{ }^{\text {out }} \mathrm{ALP} 16$ peptide gives rise to an isotropic signal (I), which most likely represents a cubic lipid phase $(21,25) . \mathrm{W}_{2}$ in ALP16 thus behaves in a fashion similar to that of WALP16, whereas $\mathrm{W}_{2}{ }^{\text {out } A L P 16}$ resembles the behavior of longer WALP peptides such as WALP17 and WALP19 (21). Because the preference for a specific nonlamellar phase is believed to reflect the difference in minimal hydrophobic thickness for each of these phases (21), these results demonstrate that the hydrophobic matching criterium, or effective peptide length, for WALP analogues in relatively thick lipid bilayers is the distance between the innermost flanking Trp residues.

Lipid Bilayer Perturbations by Relatively Long TrpFlanked Peptides. Under positive mismatch conditions, lipid bilayers respond to incorporation of (relatively long) WALP peptides by slightly ordering the lipid acyl chains (20). To investigate whether in this case it is the total length of these 
Table 1: Amino Acid Sequences of the WALP Peptides Used and the Calculated Length of the Leucine-Alanine (LA) Stretch, When Present as an $\alpha$-Helix ${ }^{a}$

\begin{tabular}{|c|c|c|}
\hline peptide & sequence & length of LA stretch $(\AA)$ \\
\hline WALP16 & Ac-GWWLALALALALAWWA-Etn & 15.0 \\
\hline $\mathrm{W}_{2}$ in ALP16 & Ac-GAWLALALALALAWAA-Etn & 15.0 \\
\hline $\mathrm{W}_{2}{ }^{\text {out }} \mathrm{ALP} 16$ & Ac-GWLALALALALALAWA-Etn & 18.0 \\
\hline WALP17 & Ac-GWWLALALALALALWWA-Etn & 16.5 \\
\hline WALP19 & Ac-GWWLALALALALALALWWA-Etn & 19.5 \\
\hline WALP23 & Ac-GWWLALALALALALALALALWWA-Etn & 25.5 \\
\hline WALP $^{16} 26$ & Ac-GAGAGAWWLALALALALAWWAGAGAG-Etn & 15.0 \\
\hline WALP27 & Ac-GWWLALALALALALALALALALALWWA-Etn & 31.5 \\
\hline KALP23 & Ac-GKKLALALALALALALALALKKA-amide & 25.5 \\
\hline KALP31 & Ac-GKKLALALALALALALALALALALALALKKA-amide & 37.5 \\
\hline
\end{tabular}

${ }^{a}$ It is assumed that each amino acid has a length of $1.5 \AA$.
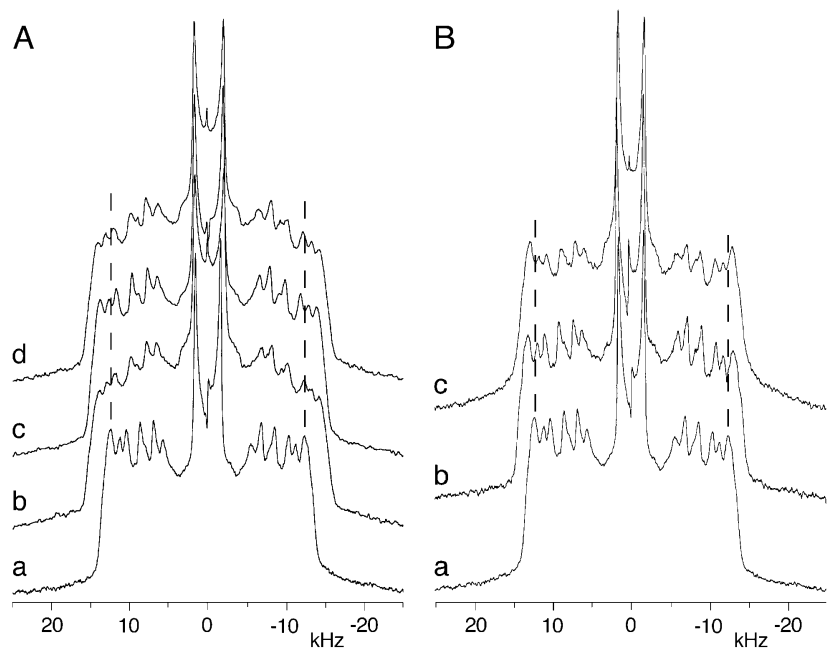

FIGURE 2: ${ }^{2} \mathrm{H}$ NMR spectra of dispersions of 14:0-PC- $d_{27}$ in the absence (a) and presence of the following peptides at a 1:30 peptide/ lipid molar ratio: (A) WALP1626 (b), WALP16 (c), and WALP23 (d); (B) KALP31 (b) and KALP23 (c).

hydrophobic peptides or the inter-tryptophan distance that determines the extent of lipid chain ordering, we compared the effect of WALP16 with that of WALP ${ }^{16} 26$, a Trp-flanked peptide of 26 hydrophobic residues with the flanking residues at a position similar to that in the shorter WALP16 analogue (see Table 1). WALP ${ }^{16} 26$ was incorporated at a 1:30 peptide/ lipid molar ratio into bilayers of 14:0-PC with perdeuterated $s n-2$ chains, and the effect of the peptide on 14:0-PC acyl chain order was characterized by ${ }^{2} \mathrm{H}$ NMR. Figure $2 \mathrm{~A}$ shows that relative to the ${ }^{2} \mathrm{H}$ NMR spectrum of pure 14:0-PC (spectrum a), incorporation of WALP ${ }^{16} 26$ (spectrum b) increases the quadrupolar splittings for the central methyl and the outer methylene peaks, indicating that the peptide increases lipid acyl chain order. Comparison with the previously obtained spectra in the presence of WALP16 and WALP23 (spectra $\mathrm{c}$ and d) indicates that the acyl chain ordering effect of WALP ${ }^{16} 26$ is similar to the effect of WALP16 and significantly smaller than that of WALP23. This is not due to a different extent of incorporation, because CD spectra of WALP ${ }^{16} 26$ are indicative of a fully associated transmembrane $\alpha$-helix (data not shown), as previously demonstrated for other peptide-14:0-PC systems described in this study $(21,22,25)$. The effects on bilayer thickness were estimated as described under Methods, with the results given in Table 2. On the basis of these calculations, the WALP ${ }^{16} 26$-induced increase in mean 14:0-PC bilayer thickness is $0.4 \AA$, which is indeed the same as the increase caused
Table 2: Changes in Mean Hydrophobic Thickness $(\Delta d)^{a}$ Relative to the Thickness of Pure $s n-2$ Chain Perdeuterated 14:0-PC $(22.5$ $\AA^{b}$ ) for Systems at a 1:30 Peptide/Lipid Molar Ratio ${ }^{c}$

\begin{tabular}{cccc}
\hline peptide & $\Delta d(\AA)$ & peptide & $\Delta d(\AA)$ \\
\hline WALP16 & $+0.4^{b}$ & KALP23 & $+0.2^{b}$ \\
WALP19 & $+0.6^{b}$ & KALP31 & +0.3 \\
WALP23 & $+1.0^{b}$ & & \\
WALP1626 & +0.4 & & \\
\hline
\end{tabular}

${ }^{a}$ Estimated from ${ }^{2} \mathrm{H}$ NMR-derived order parameters as outlined under Materials and Methods. ${ }^{b}$ Value obtained from ref 22. ${ }^{c}$ Measurements were performed at $34{ }^{\circ} \mathrm{C}, 10{ }^{\circ} \mathrm{C}$ above the main phase transition temperature of the pure 14:0-PC. The estimated experimental precision in hydrophobic thickness is $\pm 0.1 \AA$.

by the shorter WALP16 peptide. This again indicated that the distance between the flanking Trp residues is critical for determining the extent of mismatch, suggesting that the lipid rearrangements are directed to keep the Trp residues at a defined (preferred) position in the membrane.

Membrane Location of Trp Residues under Mismatch Conditions. Solid-state ${ }^{1} \mathrm{H}$ MAS NMR methods allow for investigation of the membrane location of Trp residues in different WALP-PC combinations by exploiting the ring current effects of the Trp indole side chain, which induces changes in the chemical shifts of lipid protons located in close proximity (e.g., ref 32). As indicated in Figure 3, the ${ }^{1} \mathrm{H}$ MAS NMR resonances of the different moieties of 14: 0 -PC are resolved in the absence and presence of peptides, except for the chemically homogeneous chain methylenes, that is, $\mathrm{C}^{4} \underline{\mathrm{H}}_{2}-\ldots-\mathrm{C}^{13} \underline{\mathrm{H}}_{2}$. Because the 14:0-PC lipids are short, they maintain a bilayer organization in the presence of the WALP peptides, as was confirmed by ${ }^{31} \mathrm{P}$ NMR measurements (data not shown). As shown in Figure 4, incorporation of WALP16, WALP19, or WALP23 results in significant upfield chemical shift changes relative to pure 14:0-PC, especially for the resonances corresponding to the lipid acyl chain and to the majority of the choline protons. These effects seem to increase with the length of the peptides. In contrast, a Lys-flanked KALP23 analogue (see Table 1) induces significant chemical shift changes of only the acyl chain peaks, which cannot result from aromatic ring current effects and apparently reflect a general effect on the average chain conformation due to the presence of a transmembrane polyleucine-alanine helix. Thus, in the presence of all three WALP analogues, large Trp-specific effects are observed on the $\mathrm{C}^{2} \mathrm{H}_{2}$ and $\mathrm{C}^{3} \mathrm{H}_{2}$ methylenes and on the $\mathrm{CH}_{2}(\beta)$ and $\mathrm{N}\left(\mathrm{CH}_{3}\right)_{3}$ protons of the choline group, indicating that these moieties are in close proximity to the indole rings. Given that the glycerol group links the upper acyl chain segments 


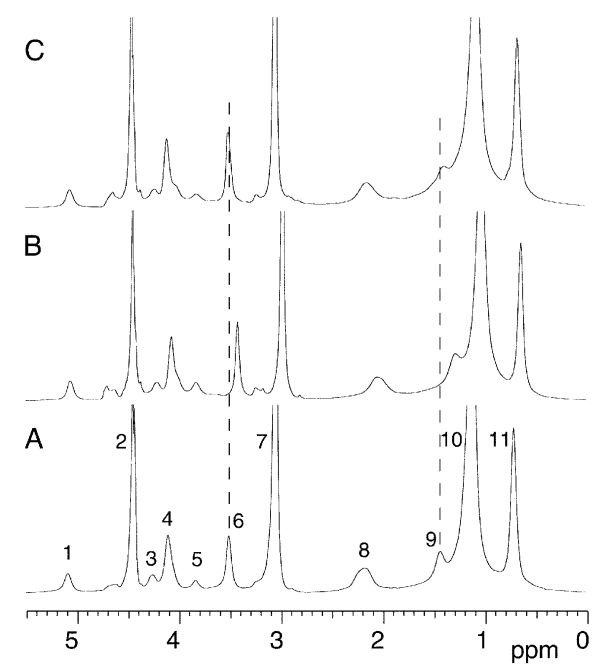

FIGURE 3: ${ }^{1} \mathrm{H}$ MAS NMR spectra of dispersions of 14:0-PC in $\mathrm{D}_{2} \mathrm{O}$, in the absence (a) and presence of WALP19 (b) and KALP23 (c). The molar ratio of peptide to lipid is 1:10. Resonances in the pure 14:0-PC spectrum were assigned according to ref 27: (1) $s n$ 2, (2) HOD, (3) sn-1, (4) $\mathrm{CH}_{2}(\alpha)$, (5) sn-3, (6) $\mathrm{CH}_{2}(\beta)$, (7) $\mathrm{N}\left(\mathrm{CH}_{3}\right)_{3}$, (8) $\mathrm{C}^{2} \underline{\mathrm{H}}_{2}$, (9) $\mathrm{C}^{3} \underline{\mathrm{H}}_{2}$, (10) $\overline{\mathrm{C}}^{4} \underline{\mathrm{H}}_{2}-\mathrm{C}^{13} \underline{\mathrm{H}}_{2}$, and (11) $\mathrm{C}^{14} \underline{\mathrm{H}}_{3}$.

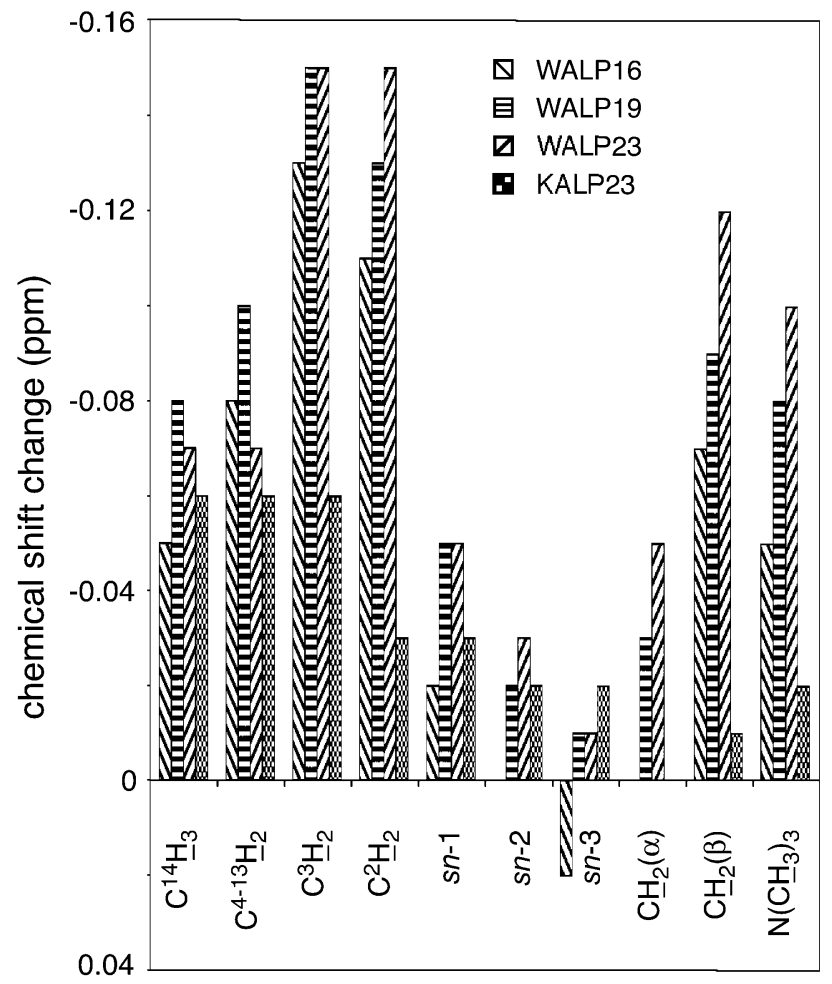

FIGURE 4: Changes in ${ }^{1} \mathrm{H}$ NMR chemical shifts of the assigned resonances, with respect to pure 14:0-PC, induced by incorporation of WALP16, WALP19, WALP23, and KALP23, as indicated.

with the choline moiety, the absence in all samples of large chemical shift changes of the $s n$-protons is counterintuitive, but from a more detailed analysis of ring current effects it follows that this perturbation pattern is consistent with a location of the Trp side chains between the upper acyl chain and choline moieties, that is, near the lipid carbonyl groups (see Discussion).

Comparison with Lys-Flanked Peptides. To gain insight into the extent to which the properties of Trp residues specifically influence peptide-bilayer interactions, we have studied analogous peptides that are flanked by residues other

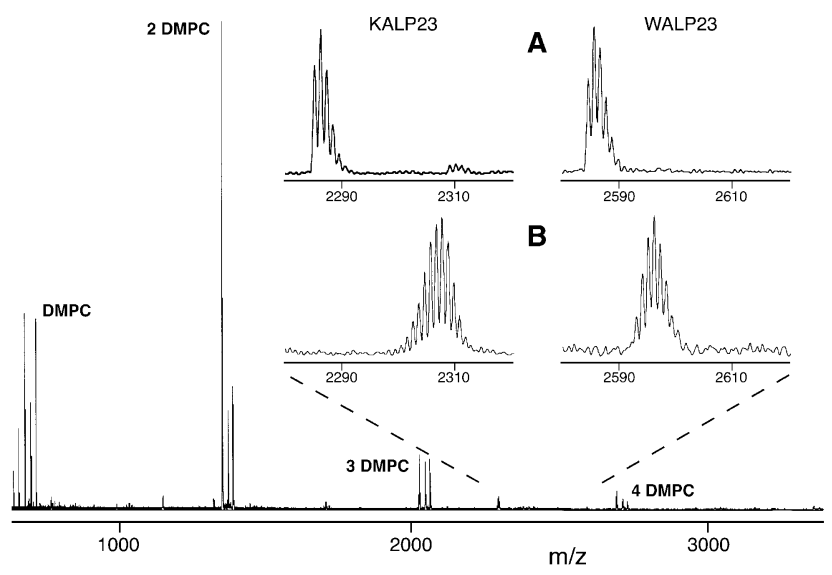

FIGURE 5: Positive mode nano-ESI mass spectrum derived from WALP23 and KALP23 in vesicles of 14:0-PC at a 1:25 peptide/ lipid molar ratio, after incubation in $98 \% \mathrm{D}_{2} \mathrm{O}$ for $2 \mathrm{~min}$. The peptides are almost exclusively detected as monomeric ions, whereas multimers as well as monomers of 14:0-PC are observed. The enlarged isotope envelopes of the partly deuterated peptides are depicted (inset B), and isotope envelopes of the nondeuterated peptides are shown for comparison (inset A).

than Trp. Our previous observation that WALP23 causes a larger increase in the apparent thickness of 14:0-PC bilayers than KALP23 (1.0 versus $0.2 \AA$ ) suggests that Trp-flanked peptides tend to avoid a positive hydrophobic mismatch with the bilayer, whereas Lys-flanked analogues do not $(22,25)$. However, it is possible that KALP23, due to different properties of its flanking residues, experiences only a very small and perhaps not even significant effective positive mismatch (22). Therefore, in the present study, the effect of the considerably longer KALP31 (Table 1) was investigated. This peptide, which has previously been shown to associate completely with 14:0-PC as a transmembrane $\alpha$-helix (23, 33 ), was incorporated in bilayers of $s n-2$ perdeuterated 14 : 0 -PC. As shown in Figure $2 \mathrm{~B},{ }^{2} \mathrm{H}$ NMR spectra in the absence and presence of KALP31 are rather similar, and order parameter analysis indicates that KALP31 causes an increase of only $\sim 0.3 \AA$ in the hydrophobic bilayer thickness (Table 2). Thus, although KALP31 has a hydrophobic stretch that is clearly too long to span the bilayer, it has a very similar effect on 14:0-PC bilayers as the substantially shorter KALP23.

Interfacial interactions of transmembrane peptides can also be investigated by analyzing the extent of their exposure to the aqueous phase. We have shown previously that mass spectrometry (MS) in combination with hydrogen/deuterium (H/D) exchange allows for determination of the positiondependent kinetics of deuterium exchange of transmembrane peptides after dilution of unilamellar vesicles into deuterated buffer $(28,29)$. Figure 5 presents a mass spectrum from simultaneously incubated and analyzed vesicles of WALP23/ 14:0-PC and KALP23/14:0-PC. The expanded spectra display the isotope envelopes of the $[\mathrm{M}+\mathrm{Na}]^{+}$ions of nondeuterated WALP23 and KALP23 (inset A), as well as those of the peptides after 2 min of exposure of the vesicles to deuterated buffer (inset B). The shifts in masses of the analogues indicate that within this short time interval, during which only the parts of a peptide that are exposed to the interface or the aqueous solution participate in H/D exchange, KALP23 takes up 19 deuterium atoms, whereas WALP23 takes up only 9 deuterium atoms. In similar experiments, 


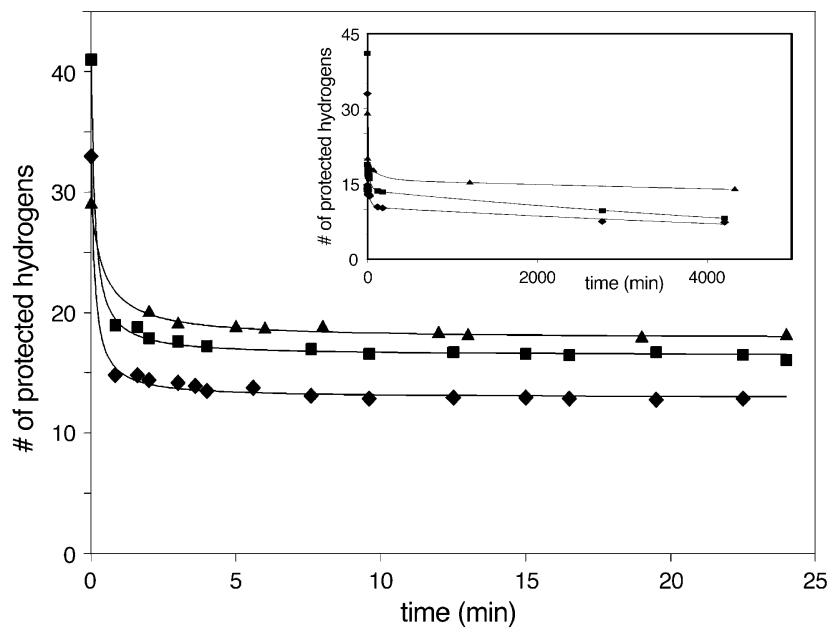

FIGURE 6: Time course for deuterium exchange, after dilution with deuterated buffer, of KALP23 (diamonds), KALP31 (squares), and WALP23 (triangles), incorporated in 14:0-PC bilayers at a 1:25 peptide/lipid molar ratio. The inset shows the time course for longer incubation times.

Table 3: Number of Labile Hydrogens and Number of Protected ${ }^{a}$ Hydrogens after 2 min and after 3 Days of Incubation in Deuterated Buffer $^{b}$

\begin{tabular}{cccc}
\hline & \multirow{2}{*}{$\begin{array}{c}\text { labile } \\
\text { peptide }\end{array}$} & \multicolumn{2}{c}{ protected hydrogens } \\
\cline { 3 - 4 } hydrogens & after 2 min & after 3 days \\
\hline KALP31 & 41 & 18 & 8 \\
KALP23 & 33 & 14 & 7 \\
WALP23 & 29 & 20 & 14 \\
WALP16 & 22 & $15^{c}$ & $8^{c}$ \\
\hline
\end{tabular}

${ }^{a}$ Protected hydrogens can be assigned to the central Leu and Ala residues $(28,29) .{ }^{b}$ The number of protected hydrogens is obtained by subtracting the number of exchanged hydrogens from the total number of labile hydrogens. ${ }^{c}$ Value obtained from ref 28.

the deuterium content of KALP31 was determined at different time intervals and compared with data from KALP23 and WALP23. Exchange data are presented in Figure 6 as the number of protected hydrogens, because the numbers of exchangeable hydrogens are different for Trp and Lys side chains (1 and 2, respectively). All three peptides undergo a fast initial H/D exchange within the first few minutes after dilution, after which time the rate of exchange levels off and the number of protected exchangeable hydrogens appears to stabilize in the order WALP23 > KALP31 > KALP23. However, a very slow exchange continues (Figure 6, inset), which is fastest for KALP31. As listed in Table 3, after 3 days, only 8 labile hydrogens of the KALP31 analogue are protected against exchange, a value that is similar to that observed for KALP23 and WALP16 but considerably less than the 14 protected hydrogens of WALP23. It should be noted that the exchange characteristics of WALP ${ }^{16} 26$ are also similar to those of WALP16 and not to longer WALP analogues $(28,29)$. The fraction of the central Leu-Ala stretch that is protected against H/D exchange thus clearly increases with the intertryptophan distance within the Trp-flanked analogues, but appears to be independent of the length of the inter-lysine distance within the Lys-flanked peptides.

\section{DISCUSSION}

Hydrophobic Matching and Interfacial Anchoring. It has been documented extensively that the extent of peptide/ bilayer hydrophobic matching influences membrane organization (e.g., refs 9 and 15-19). The design of the particular model peptides used, however, often renders it difficult to assess the importance of interfacial interactions between lipids and flanking residues. We have investigated, therefore, whether the interfacial interactions of Trp side chains are sufficiently large to dominate the effects induced by hydrophobic mismatch alone.

For two relatively short peptides, $\mathrm{W}_{2}{ }^{\text {in }} \mathrm{ALP} 16$ and $\mathrm{W}_{2}{ }^{\text {out }}$ ALP16, the effects on lipid organization demonstrate that the inter-tryptophan distance is more important than the total hydrophobic length (see Figure 1). Interestingly, $\mathrm{W}_{2}{ }^{\text {in }} \mathrm{ALP} 16$ induces the same lipid phase modulations as WALP16, whereas $\mathrm{W}_{2}{ }^{\text {out }} \mathrm{ALP} 16$ behaves as if it is effectively longer. This suggests that under negative mismatch conditions the inner Trp residues determine the effective hydrophobic length of a peptide.

Also under conditions of positive mismatch, lipid adaptations are sensitive to the positions of the Trp residues rather than to the overall hydrophobic length, as was demonstrated by the similarity of the effects of WALP16 and WALP ${ }^{16} 26$ (Table 2). These latter data are in agreement with mass spectrometric data, which suggested that both peptides have the same number of stably protected hydrogens in the transmembrane region (29). In contrast to the situation in the case of negative mismatch, mass spectrometric measurements indicate that in the case of positive mismatch the outer Trp residues in WALP peptides determine their effective protected lengths. This is based on the extent of exposure of backbone hydrogens to the aqueous environment (28). These observations can be rationalized if the Trp side chains were acting as interfacial anchors, preferring to be located within a rather narrow positional range at the membranewater interface. The indole rings resist displacement in either direction, whether to a deeper or shallower membrane location. For an energetically favorable interaction between Trp-flanked peptides and PC bilayers, it is more important to achieve "interfacial matching" than hydrophobic matching.

Preferred Membrane Position of Trp Indole Rings. Additional support for a specific interfacial localization of Trp indole rings comes from the ${ }^{1} \mathrm{H}$ MAS NMR data. The diamagnetic current in aromatic ring systems, induced by the magnetic field, causes upfield displacements of the chemical shifts of protons located up to $\sim 5 \AA$ above and below the ring, but downfield displacements for protons next to the ring (e.g., refs 32 and 34). The chemical shifts observed for 14:0-PC protons in the presence of the Lys-flanked KALP23 analogue demonstrate that the mere presence of a transmembrane peptide also causes upfield chemical shift changes for almost all of the lipid resonances, primarily those corresponding to the acyl chains (Figure 4). This effect cannot be due to aromatic ring currents and apparently reflects a general sensitivity of the lipid protons to the proximity of amino acid side chains, which is perhaps related to peptide-induced ordering of the bilayer. When the chemical shift changes induced by the WALP analogues are compared relative to those induced by KALP23, it is evident that the $\mathrm{C}^{3} \mathrm{H}_{2}$ and $\mathrm{C}^{2} \mathrm{H}_{2}$ chain methylene and $\mathrm{CH}_{2}(\beta)$ and $\mathrm{N}\left(\mathrm{CH}_{3}\right)_{3}$ headgroup protons are subject to large upfield Trpspecific changes. In contrast, downfield Trp-specific changes are apparent for the $s n-3$ glycerol proton (and in the case of WALP16 also for the $s n-1$ and $s n-2$ protons), and for 
WALP16 this displacement is downfield even relative to the chemical shift value of the $s n-3$ proton in a peptide-free 14:0-PC bilayer (Figure 4). This suggests an opposite ring current effect as observed for the upper acyl chain and choline resonances. Although interpretation of the ${ }^{1} \mathrm{H}$ NMR MAS data is complicated by factors such as the dynamic nature of the interface, the inequivalence of the different tryptophans of WALP, and the inequivalence of the $s n-1$ and $s n-2$ acyl chains, the chemical shift changes nevertheless point to a situation in which, as seen from the bilayer core, the indole side chains are preferentially located higher than the $\mathrm{C}^{3} \underline{\mathrm{H}}_{2}$ and $\mathrm{C}^{2} \underline{\mathrm{H}}_{2}$ chain methylenes, approximately at the same level as the glycerol group and below the choline moiety. For the three WALP analogues, the slightly larger Trp-specific changes of the $\mathrm{C}^{2} \underline{\mathrm{H}}_{2}$ chain protons as opposed to the choline protons suggest that the Trp side chains are located slightly closer to the former group, that is, in the lipid carbonyl region.

Within this overall chemical shift change pattern, some interesting trends can be observed. The displacement of the $\mathrm{N}\left(\mathrm{CH}_{3}\right)_{3}$ and $\mathrm{CH}_{2}(\beta)$ resonances, but not of the $\mathrm{CH}_{2}(\alpha)$ resonance, is of comparable magnitude to the chemical shift change of the $\mathrm{C}^{3} \underline{\mathrm{H}}_{2}$ and $\mathrm{C}^{2} \underline{\mathrm{H}}_{2}$ methylenes. This probably reflects an arrangement of the choline segment, which, connected to the glycerol group at the $\mathrm{CH}_{2}(\alpha)$ position, is oriented roughly parallel to the bilayer surface instead of pointing toward the aqueous phase (35). Furthermore, for the different WALP analogues, large Trp-specific effects are observed for the same lipid moieties, despite the considerable differences in peptide length, demonstrating that the Trp residues prefer to localize at a defined position within the interface. The system rearrangements required to obtain this preferential localization under positive mismatch conditions involve, as discussed, ordering of the lipid acyl chains (Table 2; see also ref 20). However, as clearly observed for the chain $\mathrm{C}^{2} \underline{\mathrm{H}}_{2}$ and choline $\mathrm{C}_{2}(\beta)$ moieties, the magnitude of the chemical shift change within the affected lipid segments does depend on WALP peptide length. This correlation indicates that the lipid acyl chain stretch does not entirely compensate for the imposed WALP/PC mismatch. Because a displacement from the preferred interfacial indole position would result in opposite changes for the upper acyl chain and choline moieties, which are not observed, we speculate that the peptide-specific modulation of chemical shift change magnitude may reflect different orientations or dynamics of the Trp side chains. Such (static or dynamic) variations of the side-chain torsion angles could result in different timeaveraged positions of the large Trp side chain with respect to the peptide backbone, thus influencing the mismatchdetermining Trp-Trp distance.

These NMR data on preferential indole location are consistent with fluorescence measurements on a large set of WALP/PC combinations, for which the Trp emission spectra are all characterized by a broad maximum around $337 \mathrm{~nm}$, typical for a relatively deep interfacial partitioning of the Trp rings (23). Also, the locations of small water-soluble compounds that mimic the Trp side chain have been investigated in model membranes using fluorescence quenching methods (12) and lipid-directed and analogue-directed NMR studies $(36,37)$. These methods confirm that membraneassociating water-soluble small Trp analogues are positioned at the polar-apolar interface close to the lipid carbonyls.
This affinity can most likely be attributed to numerous factors that give the indole ring a pronounced amphipathic character, such as dipolar interactions, imino group hydrogen bonding, and cation $-\pi$ interactions, whereas the rigidity of the aromatic ring system might also play a role $(25,36)$.

Flanking Residues and Membrane-Associating Properties of Transmembrane Peptides. For a large series of WALP analogues in a range of PC lipids, the extent of peptide incorporation correlates with the extent of peptide-bilayer mismatch (23). The observation that WALP ${ }^{16} 26$ can be fully accommodated in a 14:0-PC bilayer, whereas WALP27 cannot (23), implies that Trp-flanked peptides can associate well with PC membranes only when an interfacial position for the Trp residues can be realized on both sides of the bilayer. When the extent of "interfacial mismatch" with the bilayer is relatively small, the lipid acyl chain conformation (20), peptide tilt $(23,38)$, and local ring orientation (this study; see also ref 39) may adapt so that the Trp indole rings are located near the lipid carbonyl region.

In contrast to the behavior of Trp-flanked analogues, the extent of incorporation of Lys-flanked peptides is not reduced when the peptides are relatively long $(17,18,22)$. Even the Lys-flanked KALP31, with a hydrophobic length of at least $37.5 \AA$, fully associates with a 14:0-PC bilayer and exhibits behavior similar to that of the considerably shorter KALP23 (23). The results suggest that for relatively long Lys-flanked polyleucine/alanine peptides, the extent of peptide/bilayer hydrophobic mismatch is not of prime consideration. The general concept is in agreement with the behavior of Trpflanked peptides, for which the position of the Trp residues is more important than the length of the hydrophobic segment, under conditions of both positive and negative mismatch. When relatively short with respect to hydrophobic bilayer thickness, Lys-flanked analogues also induce compensating bilayer rearrangements (22). Unlike Trp-flanked peptides, the membrane-association properties of Lys-flanked peptides are, therefore, most likely influenced predominantly by a necessity to avoid exposure of the flanking side chains to the relatively hydrophobic membrane interior. The deepest acceptable membrane position for an uncompensated charged amino group of Lys is possibly around the lipid phosphate region (22).

Differences in the properties between Trp-flanked peptides and Lys-flanked peptides are also highlighted by H/D exchange kinetics for peptides in unilamellar vesicles. For WALP23 analogues only two more hydrogens undergo rapid exchange than for WALP16 (Table 3). After longer incubation times, the total exchange increases, but the difference in the number of protected hydrogens between the two peptides remains similar and correlates with the number of protected peptide bonds between the inner Trp residues. For the KALP peptides, no such scaling of the protected length with the inter-lysine distance was observed. Although there is a considerable difference in hydrophobic length between KALP23 and KALP31, after prolonged incubation times, they exhibit similar numbers of protected hydrogens (Table $3)$. Because this number is the same as observed for WALP16, it most likely represents the number of hydrogens that are firmly protected in an $\alpha$-helical segment, which would span an unperturbed 14:0-PC bilayer. The isotope exchange data thus support the conclusion that KALP peptides with long hydrophobic segments can be stably 
integrated into relatively thin bilayers without experiencing a significant positive mismatch.

Conclusion. Different and diverse approaches indicate that Trp indole rings of membrane-spanning peptides anchor to the polar-apolar interface. The extent of matching of the peptide hydrophobic length with the thickness of the PC bilayer is not the primary determinant for the peptide-lipid interactions in WALP systems, but rather the tendency for the Trp indole rings to be positioned near the lipid carbonyl groups. On the basis of the similar effects on lipid conformation and H/D exchange kinetics of Trp- and Tyr-flanked peptides $(25,40)$, this principle might apply also to Tyr residues. We anticipate, nevertheless, that the relative contributions of interfacial anchoring and hydrophobic (mis)match to protein-bilayer interactions will depend on the precise composition of the bilayer lipids and the transmembrane protein segment. For example, for transmembrane segments with a hydrophobicity exceeding that of a polyLeu/ Ala stretch, hydrophobic matching requirements could overrule interfacial anchor requirements, and interfacial anchor requirements may be less stringent when the flanking residues have an aromatic ring system smaller than Trp. However, the average hydrophobicity and the number of flanking aromatic residues of the WALP analogues, especially of the peptides that have only two aromatic residues in total, should approximate the composition of the transmembrane segments of a significant fraction of membrane proteins.

\section{ACKNOWLEDGMENT}

We thank Ben de Kruijff for helpful suggestions on the manuscript, Ingo Schnell for kind assistance with the MAS NMR experiments, Gerda de Korte for HPLC analysis, and Patrick van der Wel for early contributions to Figure 1.

\section{REFERENCES}

1. Doyle, D. A., Cabral, J. M., Pfuetzner, R. A., Kuo, A., Gulbis, J. M., Cohen, S. L., Chait, B. T., and MacKinnon, R. (1998) Science 280, 69-77.

2. Schulz, G. E. (1993) Curr. Opin. Cell Biol. 5, 701-707.

3. Ulmschneider, M. B., and Sansom, M. S. P. (2001) Biochim. Biophys. Acta 1512, 1-14.

4. Wallin, E., Tsukihara, T., Yoshikawa, S., Von Heijne, G., and Elofsson, A. (1997) Protein Sci. 6, 808-815.

5. Sakai, H., and Tsukihara, T. (1998) J. Biochem. 124, 1051-1059.

6. Arkin, I. T., and Brunger, A. T. (1998) Biochim. Biophys. Acta $1429,113-128$.

7. Landolt-Marticorena, C., Williams, K. A., Deber, C. M., and Reithmeier, R. A. F. (1993) J. Mol. Biol. 229, 602-608.

8. Mouritsen, O. G., and Bloom, M. (1993) Annu. Rev. Biophys. Biomol. Struct. 22, 145-171.

9. Killian, J. A. (1998) Biochim. Biophys. Acta 1376, 401-416.

10. Dumas, F., Lebrun, M. C., and Tocanne, J.-F. (1999) FEBS Lett. 458, 271-277.

11. Schiffer, M., Chang, C.-H., and Stevens, F. J. (1992) Protein Eng. $5,213-214$.

12. Kachel, K., Asuncion-Punzalan, E., and London, E. (1995) Biochemistry 34, 15475-15479.
13. Stopar, D., Spruijt, R. B., Wolfs, C. J. A. M., and Hemminga, M. A. (1996) Biochemistry 35, 15467-15473.

14. Killian, J. A., and Von Heijne, G. (2000) Trends Biochem. Sci. $25,429-434$.

15. Huschilt, J. C., Hodges, R. S., and Davis, J. H. (1985) Biochemistry 24, 1377-1386.

16. Zhang, Y.-P., Lewis, R. N. A. H., Henry, G. D., Sykes, B. D., Hodges, R. S., and McElhaney, R. N. (1995) Biochemistry 34, $2348-2361$.

17. Ren, J., Lew, S., Wang, J., and London, E. (1999) Biochemistry $38,5905-5912$.

18. Webb, R. J., East, J. M., Sharma, R. P., and Lee, A. G. (1998) Biochemistry 37, 673-679.

19. Harzer, U., and Bechinger, B. (2000) Biochemistry 39, 1310613114.

20. De Planque, M. R. R., Greathouse, D. V., Koeppe, R. E., II, Schäfer, H., Marsh, D., and Killian, J. A. (1998) Biochemistry 37, 9333-9345.

21. Killian, J. A., Salemink, I., De Planque, M. R. R., Lindblom, G., Koeppe, R. E., II, and Greathouse, D. V. (1996) Biochemistry 35, 1037-1045.

22. De Planque, M. R. R., Kruijtzer, J. A. W., Liskamp, R. M. J., Marsh, D., Greathouse, D. V., Koeppe, R. E., II, De Kruijff, B., and Killian, J. A. (1999) J. Biol. Chem. 274, 20839-20846.

23. De Planque, M. R. R., Goormaghtigh, E., Greathouse, D. V., Koeppe, R. E., II, Kruijtzer, J. A. W., Liskamp, R. M. J., De Kruijff, B., and Killian, J. A. (2001) Biochemistry 40, 50005010 .

24. Greathouse, D. V., Goforth, R. L., Crawford, T., Van der Wel, P. C. A., and Killian, J. A. (2001) J. Pept. Res. 57, 519-527.

25. De Planque, M. R. R., Boots, J.-W. P., Rijkers, D. T. S., Liskamp, R. M. J., Greathouse, D. V., and Killian, J. A. (2002) Biochemistry $41,8396-8404$

26. Weiss, T. M., Van der Wel, P. C. A., Killian, J. A., Koeppe, R. E., II, and Huang, H. W. (2003) Biophys. J. 84, 379-385.

27. Forbes, J., Bowers, J., Shan, X., Moran, L., Oldfield, E., and Moscarello, M. A. (1988) J. Chem. Soc., Faraday Trans. 1 84, $3821-3849$.

28. Demmers, J. A. A., Van Duijn, E., Haverkamp, J., Greathouse, D. V., Koeppe, R. E., II, Heck, A. J. R., and Killian, J. A. (2001) J. Biol. Chem. 276, 34501-34508.

29. Demmers, J. A. A., Haverkamp, J., Heck, A. J. R., Koeppe, R. E., II, and Killian, J. A. (2000) Proc. Natl. Acad. Sci. U.S.A. 97, 3189-3194.

30. Seelig, J. (1978) Biochim. Biophys. Acta 515, 105-140.

31. Cullis, P. R., and De Kruijff, B. (1979) Biochim. Biophys. Acta 559, 399-420.

32. Case, D. A. (1995) J. Biomol. NMR 6, 341-346.

33. Kol, M. A., De Kroon, A. I. P. M., Rijkers, D. T. S., Killian, J. A., and De Kruijff, B. (2001) Biochemistry 40, 10500-10506.

34. Stamm, H., and Jäckel, H. (1989) J. Am. Chem. Soc. 111, 65446550 .

35. Seelig, J., Macdonald, P. M., and Scherer, P. G. (1987) Biochemistry 26, 7535-7541.

36. Yau, W.-M., Wimley, W. C., Gawrisch, K., and White, S. H. (1998) Biochemistry 37, 14713-14718.

37. Persson, S., Killian, J. A., and Lindblom, G. (1998) Biophys. J. $75,1365-1371$.

38. Van der Wel, P. C. A., Strandberg, E., Killian, J. A., and Koeppe, R. E., II (2002) Biophys. J. 83, 1479-1488.

39. Koeppe, R. E., II, Hatchett, J., Jude, A. R., Providence, L. L., Andersen, O. S., and Greathouse, D. V. (2000) Biochemistry 39, 2235-2242.

40. Demmers, J. A. A., Rijkers, D. T. S., Haverkamp, J., Killian, J. A., and Heck, A. J. R. (2002) J. Am. Chem. Soc. 124, 11191-11198.

\section{BI027000R}

